\title{
Stimulus modality and short-term memory in rats
}

\author{
JULIA WALLACE \\ Princeton University, Princeton, New Jersey 08540
}

and

\author{
PAMELA A. STEINERT, STANLEY R. SCOBIE, and NORMAN E. SPEAR \\ State University of New York, Binghamton, New York 13901
}

\begin{abstract}
Two experimental paradigms are presented aimed at determining the retention of auditory and visual information over brief delay intervals. First, a conditional delayed matching-tosample procedure was used in which rats were required to symbolically match the modality of the sample stimulus with one of two comparison stimuli. In the second experiment, subjects were trained and tested using a Konorski-type procedure. Despite the conceptual and procedural differences between the two procedures, subjects in both experiments showed steeper forgetting functions for visual events than for auditory events, while performance levels at 0-sec delay intervals were equivalent for both stimuli. These results, when taken together with related research conducted with pigeons, suggest that content of memory may have important influences on the short-term retention abilities of animal subjects.
\end{abstract}

Surprisingly little is known about forgetting of recent events in the laboratory rat. Although Hunter (1913) reported that rats could retain the spatial location of visual stimulus representing food for a maximum of $10 \mathrm{sec}$, successful performance appeared to be dependent upon the use of response-directing behaviors which mediated the delay. More recent investigators have modified Hunter's procedure and have studied short-term retention using delayed response and delayed alternation tasks (e.g., Gordon, Brennan, \& Schlesinger, 1976; Roberts, 1974). These paradigms have been problematic, however, primarily because they do not allow for precise experimental specification of the event the rat is required to retain. While the delayed matching-to-sample (DMTS) task has proven to be a useful method for measuring short-term retention processes in the pigeon (Roberts \& Grant, 1976), monkey (D'Amato, 1973), dolphin (Herman \& Gordon, 1974) and goldfish (Steinert, Fallon, \& Wallace, Note 1), no comparable paradigm has been available for the study of these same mechanisms in the rat. The need for such tests to be devel-

This research was supported by a Public Health Service predoctoral fellowship, No. 05075, NIA Postdoctoral Fellowship AG05088, and a Sigma Xi grant-in-aid of research to the first author, grants from the National Science Foundation (BMS 7424191 and BNS 78-02360) to the fourth author, and Grant 5-R01MH 27210 from the National Institute of Mental Health and funds from the Office of Research Development, State University of New York at Binghamton, to the third author. We would like to thank Diane Feldman, Gregory Smith, and Norman Richter for assistance in various aspects of this research. Requests for reprints should be directed to the first author, Department of Psychology, University of Northern Iowa, Cedar Falls, Iowa 50613. oped for rats is especially important because so much complementary knowledge is available about other behavioral and physiological aspects of learning and retention in this animal.

In the present research, two experimental procedures which have proven useful for the investigation of shortterm memorial processes in the rat were used. Both paradigms involve modifications of the DMTS procedure, the first using a conditional discrimination (D'Amato \& Worsham, 1974) and the second a sequential procedure originally described by Konorski (1959) and recently used successfully by Shimp and Moffit (1977) and Wasserman (1976).

Because the rat is not a "visual" animal in the sense that a variety of visual displays can be used effectively as to-be-remembered items, the present research employed brief presentations of auditory and visual stimuli as sample events. In the course of developing these tests, however, a striking finding became apparent. The auditory events chosen for retention testing were forgotten more slowly than the visual events. This result is of special interest for two reasons. First, task variables-specific aspects of the episode to be remembered (the "content of memory")- have not frequently been found to influence the rate of forgetting observed in humans or animals (Spear, 1978; but see D'Amato, 1973, pp. $240-242$, for a discussion of modality-specific retroactive interference effects). Second, our general finding with rats of less rapid forgetting of auditory than of visual events parallels, at least superficially, the "modality effect" that has been studied thoroughly with human subjects (cf. Crowder, 1976). 


\section{EXPERIMENT 1}

In the first experiment, rats were trained on an operant conditional discrimination task similar to the delayed conditional matching task used by D'Amato and Worsham (1974) and the symbolic matching task used by Carter and Eckerman (1975). The conditional nature of the discrimination required the rat to learn one response rule if an auditory stimulus was presented and another response rule if a visual stimulus was the to-be-remembered event. The advantages of this procedure over the more traditional DMTS tasks are, first, that the procedure can be adapted for use with rats because the to-be-remembered events can vary in modality, thus increasing the range of stimuli applicable for testing, and second, that differential retrieval cues are not available to the subject at the time of test.

\section{Method}

Subjects. Five experimentally naive, male, Sprague-Dawley rats obtained from Charles River Breeding Laboratories served as subjects. At the start of training, all animals were approximately 60 days of age, and were reduced to $85 \%$ of their free-feeding weights. Since the training of these rats was conducted over a long duration of time, their individual weights were increased by $5 \mathrm{~g}$ per week until they were approximately 250 days of age. This increase, based on the weight gain of nondeprived SpragueDawley rats of similar ages maintained in this laboratory, kept deprivation levels constant throughout the duration of the study.

Apparatus. All rats were trained in a single three-lever operant chamber, measuring $39.5 \mathrm{~cm}$ long, $35.0 \mathrm{~cm}$ wide, and $28.0 \mathrm{~cm}$ high. On the center of the aluminum front wall and $1.5 \mathrm{~cm}$ above the steel-bar floor was a 4-cm-diam opening which allowed access to a .1-ml dipper cup. Two retractable levers were mounted $7.5 \mathrm{~cm}$ on either side of this opening and $7.0 \mathrm{~cm}$ above the floor. A third retractable lever was centered on the aluminum rear wall $7.0 \mathrm{~cm}$ above the floor. A white-jeweled lamp fixture, $1.4 \mathrm{~cm}$ in diameter, was located $5.5 \mathrm{~cm}$ above each lever. The two sides and the ceiling of the chamber were made of clear acrylic plastic.

The chamber was housed in a dark, sound-attenuating chest with an ambient noise level of $55 \mathrm{~dB}$. Visual samples consisted of the onset of a 7.5-W bulb, located above the ceiling and providing diffuse illumination within the chamber through a sheet of white translucent plastic covering the ceiling. Presentations of auditory samples consisted of the onset of a 82-dB white noise through a $10-\mathrm{cm}$-diam speaker located in the center of the ceiling of the chamber. Experimental events were controlled automatically by electromechanical equipment located in an adjacent room. Sequencing of trials was programed by means of an eightchannel paper-tape reader.

Procedure. Upon reaching an $85 \%$ deprivation level, all rats were trained to press the levers. In the first session, a .1-ml $16 \%$ sucrose solution was initially delivered on a variable-time 1 -min schedule. The duration of access to the dipper was $10 \mathrm{sec}$ at the beginning of the session but was reduced to $4 \mathrm{sec}$ when responding began. Each response on either of the front levers was reinforced; the rear lever was retracted and unavailable. When the rat began to respond, free reinforcements were no longer delivered. After a rat had made a total of 100 responses, it was removed from the chamber; all rats met this requirement within a 1-h session. On the following day, the animals were allowed to respond freely on both levers until 100 reinforcements had been delivered. In order to prevent position biases, a lever was retracted if more than 20 consecutive responses were made, and the rat was forced to switch to the alternate lever. The 3rd day of training consisted of 96 random right and left, single, front-lever presentations. On half of these presentations, the cue light above the available lever was lit. Immediately following a response, the lever was retracted and reinforcement delivered. The interval between the 96 lever presentations was $2 \mathrm{sec}$. Training to press the single lever on the rear wall began $c$ : the 4th day. Illumination of the cue light above the rear lever as coincident with its presentation. Following a single press . 's lever one of the front levers was presented (in sequences ..... . ical to those given on Day 3 ); a response on the front le $\cdots$, produced the reinforcement and reinstituted the sequence.

After the rat learned to respond consistently on all three levers, there were four training phases. The phases were progressive in difficulty and designed to facilitate the acquisition of the conditional discrimination needed for the test of short-term retention. Transitions between phases were dependent upon the subjects' reaching an $80 \%$ correct response level for both auditory and visual stimuli in the previous phase. Phases 1 through 3 consisted of simultaneous trials; during Phase 4,0 -sec trials were introduced.

At the beginning of each trial, the rear lever was inserted into the chamber and the cue light above this lever was turned on. A single response retracted the lever, terminated the cue light, and presented either an auditory or visual stimulus. These sample stimuli were $4 \mathrm{sec}$ in duration. In the simultaneous condition, the choice stimuli (onset of one of the two front-lever cue lights) were presented $2 \mathrm{sec}$ following the onset of the sample stimulus and thus overlapped with the sample stimulus for $2 \mathrm{sec}$. In the $0-\mathrm{sec}$ condition, the choice stimuli were presented immediately after the sample stimulus had terminated. The choice stimuli always preceded the introduction of the front levers into the chamber by $2 \mathrm{sec}$ and terminated with the retraction of the front levers when a response was made.

For three rats, the front lever with the light above it (lit lever) was correct when the sample stimulus was auditory and the nonlit lever was correct when the sample stimulus was visual. These reinforcement conditions were reversed for the remaining two rats. Sucrose reinforcement immediately followed a correct response and a 20 -sec time-out followed an error. The interval between reinforcement or completion of the time-out period and the beginning of the next trial was $30 \mathrm{sec}$. The chamber was dark during these intervals and during subsequently presented delay intervals.

During Phase 1 , blocks of either auditory or visual discrimination trials were preceded by single-lever, forced-correct trials. The number of these single-lever trials was reduced during Phase 2 such that by the completion of Phase 2 the rats had received a block of 50 auditory discrimination trials followed by a block of 50 visual discrimination trials (or the reverse sequence). In Phase 3 , the number of alternations between auditory and visual trial blocks was gradually increased, but the number of trials within each daily session remained at 100 . Upon the completion of Phase 3, auditory and visual trials were presented randomly.

Introduction of a $0-\mathrm{sec}$ delay between the termination of the sample stimulus and the onset of the choice stimuli was gradually introduced during Phase 4 . Each daily session in this phase contained 48 trials; the spatial location of the choice stimuli and the presentation of an auditory or visual sample stimulus occurred randomly. The number of 0 -sec trials was gradually increased while the number of simultaneous trials was decreased until, at the end of Phase 4, all were 0 -sec trials.

Training involving greater than 0-sec delays began in Phase 5. All rats received 48 trials during each daily session. Of these trials, 36 had $0-\mathrm{sec}, 6$ had 2 -sec, and 6 had 4 -sec delays. The delay was inserted between the offset of the sample stimulus and the onset of the choice stimuli. An equal number of auditory and visual sample stimuli were presented within each session. There were four different trial sequences presented in a scrambled 
order across days. All sequences began with at least four trials at the 0-sec delay; 2- and 4-sec delay trials never occurred in succession. Counters recorded correct and incorrect responses to both auditory and visual stimulus presentations at each delay interval. The number of responses occurring on the right or left lever were recorded as were the number of correct and incorrect responses within the first or second half of each session. These later measures were collected in order to determine whether any position biases had developed over sessions and whether there was a warm-up effect within sessions.

\section{Results}

On the final day of Phase 4 training, the average probability of a correct response on the 0 -sec delay trials did not differ for auditory or visual sample stimulus presentations. The mean probability of a correct response was .91 on auditory trials (range: .83 to 1.00 ) and .94 on visual trials (range: .88 to .96). This high level of correct responding at 0 -sec delays was maintained throughout the 50 days of Phase 5 when 2-and 4-sec delays were introduced (mean correct response probability for 0-sec delays: auditory trials $=.92$, range $=.82-.97$; visual trials $=.91$, range $=.86-.94$ ). An analysis of variance conducted over all 0-sec delay trials during Phase 5 revealed no effect of sample stimulus modality, no change in response accuracy over blocks of trials, and no interaction between these variables. It should be recalled that within each daily session there were $360-\mathrm{sec}$ delay trials out of a total of 48 trials. This large 0 -sec trial proportion probably contributed to the stability of performance levels.

In Figure 1, the probability of a correct response is plotted for all delay intervals across the five blocks of 10 sessions during Phase 5. The figure indicates that there was a pronounced difference in the ability of the rats to retain stimulus information over 2and 4-sec delay intervals, depending upon the modality of the sample stimulus. Separate analyses of var-

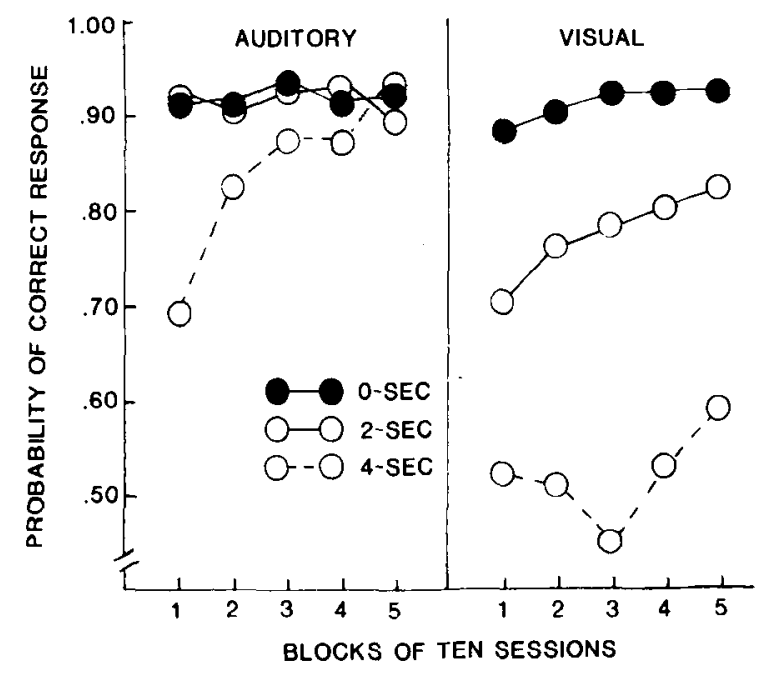

Figure 1. Probability of a correct response in each of five blocks of 10 sessions for three delay intervals.

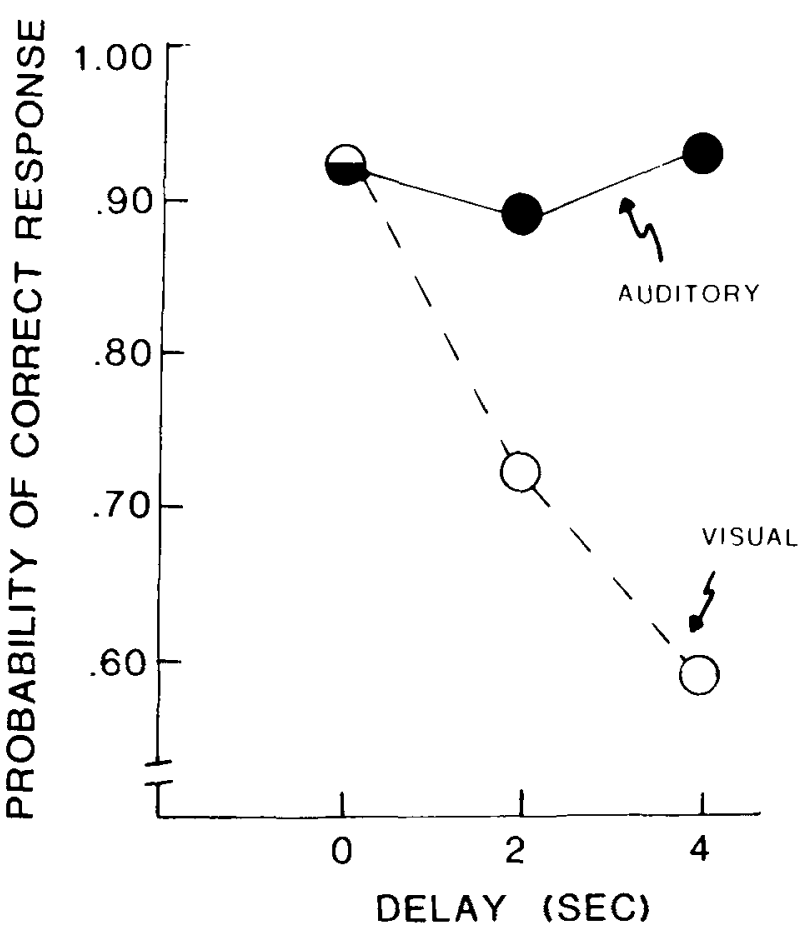

Figure 2. Probability of a correct response across delay intervals on the final block of 10 sessions.

iance performed over all blocks of trials within each modality revealed that when auditory trials were presented there was no overall effect of delay interval $(\mathrm{F}<1.0)$. There was, however, a blocks-of-trials effect $[F(4,16)=3.41, p<.05]$, as well as a Delay by Blocks-of-Trials interaction $[F(8,32)=3.03$, $\mathrm{p}<.05]$. These significant effects were due to the gradual increase in response accuracy on the 4-sec delay trials over blocks of sessions. When visual trials were statistically analyzed, the only significant effect found was the delay interval $[\mathrm{F}(2,8)=13.78$, $\mathrm{p}<.01$ ]; there was no effect of trial blocks or an interaction between these variables [Fs $<1.0$ ).

Since rats were counterbalanced as to whether a nonlit lever was correct following an auditory or a visual sample stimulus, a between-groups analysis of variance was conducted comparing the delay performance of rats within these two conditions. We thought it possible that the conditional discrimination might have been easier if a nonlit lever was correct following an auditory trial and a lit lever was correct following a visual trial. However, none of the expected interactions approached significance.

In Figure 2, the probability of a correct response on the last block of 10 sessions is plotted for both sample stimuli across the three delay intervals. The interaction between modality of the sample stimulus and delay interval is readily apparent. The probability of a correct response at the 0-sec delay, however, is identical for both auditory and visual signals. At longer delays, there is no apparent change in 
accuracy following an auditory sample stimulus, but a clear retention decrement following visual presentations. A within-subjects analysis of variance on the last trial block of 10 sessions revealed a significant effect of stimulus modality $[F(1,4)=19.72, p<.01]$, delay $[\mathrm{F}(2,8)=4.81, \mathrm{p}<.05]$, and a Modality by Delay interaction $[F(2,8)=6.66, p<.05]$.

Occasional observations of the rats during the sessions revealed no evidence of mediational behavior during the delay interval for four of the five rats. Typical behavior following a rear-leverpress consisted of an observational type of response (e.g., rearing), toward the ceiling of the chamber followed by an approach to the front of the chamber. In one subject, an auditory sample stimulus presentation was always followed by an immediate movement to the right side fo the chamber. Following a visual stimulus, a movement to the left side of the chamber was dominant, but approaches to the middle or right side were also observed. On visual delay trials, there was no dominant response; that is, this rat approached the right, left, and center equally often. In fact, correct responding on $4-\mathrm{sec}$ visual delay trials was generally inferior for this rat than for any of the others, suggesting that the presence of the mediating behavior was not facilitating the retention of the sample stimulus.

\section{Discussion}

The results of the present experiment show that rats' forgetting of a visual signal can be significantly greater than its forgetting of an auditory signal. At 0 -sec delays, conditional discrimination accuracy was identical for both auditory and visual events. However, with increasing delays, retention of the visual sample showed a marked decline, whereas no decline was evident following an auditory stimulus presentation.

Because of the nature of the conditional discrimination task, it is important to note that once the sample stimulus has been presented, the rat, ideally, need only retain the response rule corresponding to the modality of the signal. For example, when a visual stimulus is presented, the rat need only remember to respond to the lit or nonlit lever according to the acquired rule. It could be argued, therefore, that the present results merely demonstrate a more rapid forgetting of the visual response rule than the auditory response rule, modality of the sample having no affect. It does not appear that this interpretation is justified. The rats were counterbalanced with regard to the response rule associated with each of the sample events, and for all rats visual events were forgotten more rapidly than auditory events. In addition, if it is possible that visual events are less transcribable into response rules than are auditory events, this difference should have resulted in a reduction in discrimination accuracy for the visual signal at 0 -sec delay intervals.

The significance of the present results must be somewhat tempered due to the asymmetry of the research design. Although both visual and auditory stimuli were presented as sample stimuli, only visual stimuli were used as comparison stimuli. It is conceivable that the increases in chamber illumination that occurred both during the presentation of the visual sample and during the choice period were sufficiently similar to cause proactive interference effects. The tone, occurring only as the sample stimulus, would not have been similarly affected and therefore would appear to be more easily retained. This tentative explanation is supported by data from monkeys showing that visual events, but not auditory events, occurring during the delay interval can retroactively interfere with the retention of a visual signal (Worsham \& D'Amato, 1973).

The finding here that modality may influence the rat's ability to retain events over brief intervals should not overshadow the fact that the conditional discrimination procedure has been shown to be a useful tool for the assessment of short-term retention in the rat. Although the present procedure was extremely lengthy, it is probable that both the number of phases and the requirements for transitions between phases was overly conservative. D'Amato and Worsham (1974) reported that the delayed conditional matching procedure produced unstable performance levels when retention intervals were varied. With extensive pretraining, however, monkeys (D'Amato \& Worsham, 1974) and rats, as shown in the present study, are capable of achieving stable baseline performance levels on a conditional matching task.

It should be noted that in pigeons the symbolic or conditional matching-to-sample task has been found to be as easily acquired as the more traditional matching-to-sample procedure (Carter \& Eckerman, 1975). The slower acquisition occasionally found with the symbolic procedure (e.g., Eckerman, 1970) has been reported by both Carter and Eckerman (1975) and Cohen, Looney, Brady, and Aucella (1976) to be due to the discriminability of the sample stimuli used as well as the discriminability of the comparison stimuli used. For example, Carter and Eckerman (1975) reported that, regardless of the sample stimuli (line orientation or color), acquisition was faster with color comparisons than with orientation comparisons. Likewise, regardless of comparison stimuli, acquisition was superior when color stimuli rather than line stimuli were used as the sample events. For the present experiment, this interpretation would suggest that the nature of the comparison stimuli used (lit or nonlit levers) should not interact with the modality (auditory or visual) of the sample stimulus. Since we found no 
difference between rats required to match on the basis of lever illumination and sample modality, our results are consistent with this interpretation.

The present results also indicate that rats, like monkeys (D'Amato, 1973), show improvement in retention with increased practice. This result suggests that declines in retention cannot be explained by a simple, biologically inevitable process such as decay, since its occurrence is subject to modification through experience.

\section{EXPERIMENT 2}

Because the training regimen required for the conditional discrimination procedure was exceedingly lengthy, and because we were interested in determining if the differences in auditory and visual retention were specific to the paradigm or training procedure used, Experiment 2 was conducted, based on a procedure developed by Konorski (1959) and recently used successfully in pigeons by Shimp and Moffit (1977) and Wasserman (1976). The paradigm is best described as a sequential, matching-to-sample task. Rats are presented two consecutive stimuli in an operant chamber. A lever is introduced only during the presentation of the second stimulus. If the two stimuli presented are the same, a leverpress provides for the delivery of a food pellet. If the stimuli are different, no reinforcement follows a response. This paradigm allows a delay to be inserted between the offset of the first stimulus and the onset of the second. In these instances, the rat is required to remember the first so it can be compared with the second. Unlike the conditional discrimination task used in the first experiment, but similar to the more traditional DMTS procedures, this paradigm provides maximum retrieval information at the time of test. Furthermore, because of the sequential nature of the task, it is again possible to use stimulus events that vary across modalities.

\section{Method}

Subjects. The subjects were 10 6-month-old male Fischer 344 rats. The rats were obtained from Charles River breeding laboratories along with older rats of the same strain (cf. Wallace, Krauter, \& Campbell, in press). All subjects were housed individually and reduced to $85 \%$ of their free-feeding weights before training began.

Apparatus. Three single-lever operant chambers, measuring $24.5 \times 20.2 \times 38.5 \mathrm{~cm}$ high, were used. Each chamber was housed within a sound-attenuating chest. The retractable lever was mounted on the front wall of the chamber $4.5 \mathrm{~cm}$ from the grid floor and $2.5 \mathrm{~cm}$ to the right of the food hopper opening. Constant masking noise $(55 \mathrm{~dB})$ and chamber ventilation were provided during all sessions through the use of a fan attached to the side of the chest.

Two stimulus events were used in the experiment, each $5 \mathrm{sec}$ in duration. A $72-\mathrm{dB}, 4,000-\mathrm{kHz}$ tone served as the auditory event and was delivered through a 9-cm-diam speaker located in the center of the chamber ceiling. A 15-W white light located directly above the chamber served as the visual stimulus. The chamber remained dark during both intertrial and delay intervals. Experimental conditions were controlled and sequenced by conventional electromechanical equipment located in an adjoining room.

Procedure. When the rats had reached $85 \%$ of their ad-lib weights, they were given 4 days of preliminary training. On the first 2 days, the rats were trained to barpress on a CRF schedule until they had received 100 reinforcements. On the 3 rd day, the subjects were given 48 random presentations of the auditory or visual stimulus separated by $30-\mathrm{sec}$ intervals. The lever remained in the chamber throughout the session and CRF schedule remained in effect. The final day of preliminary training was identical to Day 3, except that the lever was introduced into the chamber coincident with the onset of each auditory or visual signal.

Acquisition training began on Day 5 and consisted of 15 daily sessions, each containing 96 trials. Stimulus pairs were separated from each other by a 30-sec interval. The first $\left(S_{1}\right)$ or second $\left(\mathrm{S}_{2}\right)$ stimulus of each pair was either a 5 -sec auditory (A) or visual (V) stimulus. Four different trial types were therefore possible, AA, AV, VV, or VA. Each type was presented randomly 24 times during a single session. The separation between $S_{1}$ offset and $S_{2}$ onset was $.25 \mathrm{sec}$ (hereafter designated as $0 \mathrm{sec}$ ). The response lever was inserted into the chamber simultaneously with $S_{2}$ and retracted with $S_{2}$ offset. If both $S_{1}$ and $S_{2}$ were of the same modality, a barpress delivered a single food-pellet reinforcement. Only the first response during the $5-\sec S_{2}$ duration produced reinforcement. When $S_{1}$ and $S_{2}$ were different, a response did not produce reinforcement.

Delay training began on Day 20 and continued for 10 days for all subjects that maintained a discrimination ratio of less than .45 for the final 5 of the 15 acquisition days. The discrimination ratio was calculated by dividing the probability of a response on nonreinforced trials by the probability of a response on reinforced trials. The discrimination ratio approaches zero as the probability of a response on reinforced trials exceeds the probability of a response on nonreinforced trials. Only the first response made during the $5-\sec \mathrm{S}_{2}$ period was used in this analysis. Three delays, $0(.25 \mathrm{sec}), 2$, and $5 \mathrm{sec}$, were tested. Each type of trial at each delay was presented 8 times within a single daily session. There were four sequences of the 96 trial pairs presented in a scrambled order across the acquisition and delay training days.

\section{Results}

Seven of the original 10 subjects met the .45 discrimination ratio criterion for the last 5 days of acquisition. One subject died of unknown causes during delay training; therefore, the results for both acquisition and delay are presented for six rats.

Response probability during acquisition is shown in Figure 3 for all trial types. As is clear from this figure, acquisition for these selected subjects was rapid. At the beginning of training, probabilities were high on all trials, but response probability on nonreinforced trials gradually decreased over daily sessions. Although a slightly longer time was required to reduce response probabilities on VA trials, there were no response differences between these trials and AV trials across the last five acquisition days ( $F<1.00)$.

In Figure 4, the discrimination ratio across the three delay intervals is presented. Trials which required the retention of an auditory signal (AA and AV) are depicted separately from trials requiring the retention of a visual signal (VV and VA). Similar to the results from Experiment 1, there was no 


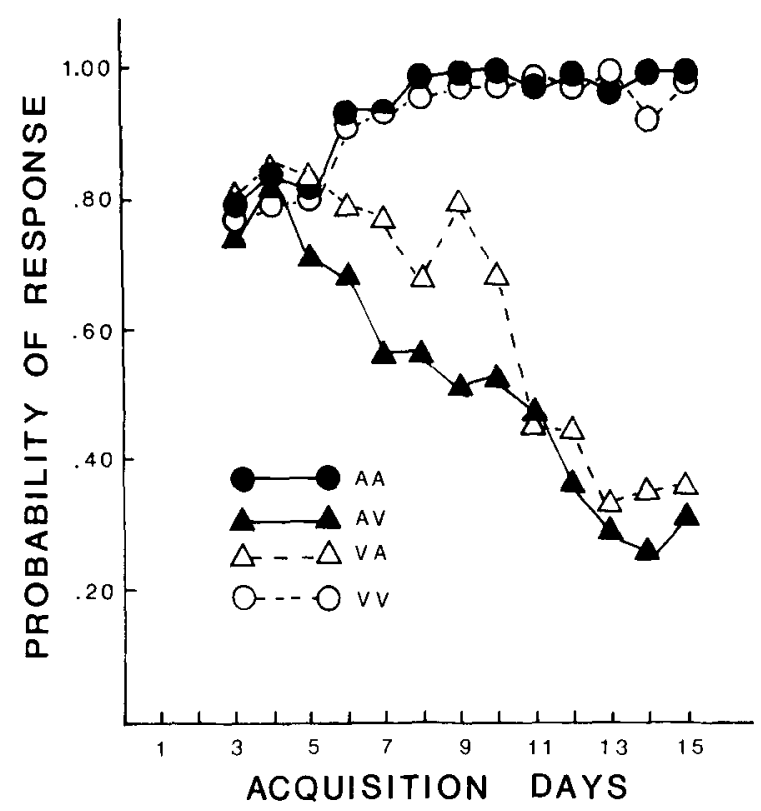

Figure 3. Probability of a response on each of four trial types across 15 days of acquisition.

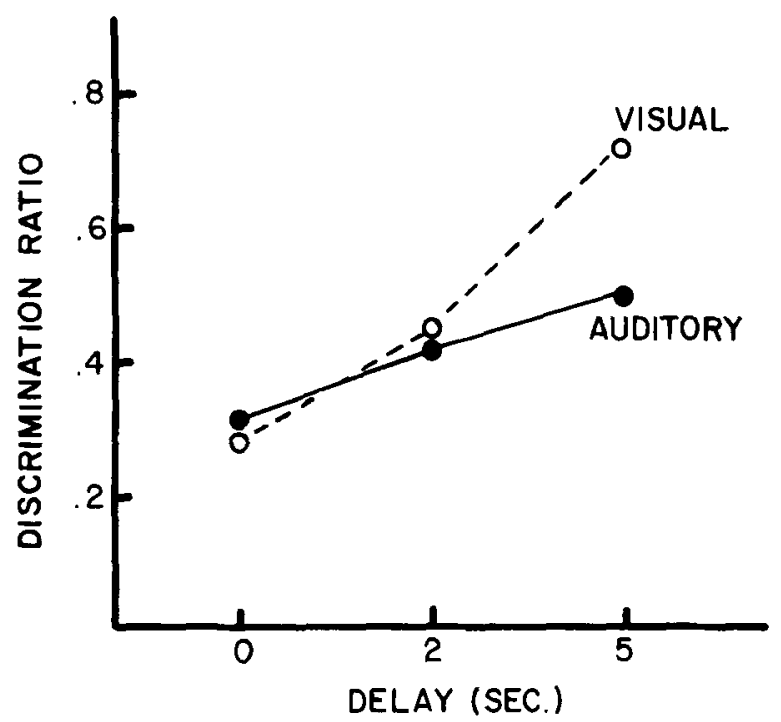

Figure 4. Discrimination ratio on trials requiring the retention of an auditory or a visual signal across delay intervals.

differential effect of modality on discrimination performance when no delay was required $(F<1.0)$. However, the rate of forgetting for visual signals was significantly greater than that of auditory signals. Both types of events, however, showed a clear retention deficit as the delay interval was increased. An analysis of variance conducted across the final 5 days of delay testing revealed a significant effect of delay $[F(2,10)=15.12, p<.001]$, and a Delay by Modality interaction $[\mathrm{F}(2,10)=7.49, \mathrm{p}<.05]$. There was no main effect of modality $[\mathrm{F}(1,5)=2.39]$. Further analyses demonstrated that the interaction resulted from a significant effect of modality which occurred only at the 5-sec delay interval $[\mathrm{F}(1,5)=$ $13.70, \mathrm{p}<.05]$.

During Days 6-10 of delay testing, the discrimination ratio was computed separately for trials occurring within the first half of each session vs. trials in the last half. It was tentatively expected that, as the number of daily trials increased, discrimination performance might be reduced due to interference from previous trial types (Keppel \& Underwood, 1962). This expectation was not supported by the data. In fact, retention at all delay intervals appeared to be slightly enhanced during the second half of each session, possibly due to a warm-up effect on early trials.

\section{GENERAL DISCUSSION}

The present experiments have described two independent procedures aimed at measuring the rats' ability to retain information over short retention intervals. These paradigms differ in significant ways. First, in the conditional matching task of Experiment 1 , rats were required to symbolically associate the modality of the sample signal with one of two comparison stimuli. Thus, as in traditional recall tests with human subjects, the sample stimuli were not available at the time of test. The Konorski task, however, was more closely related to a recognition procedure. At the time of test, the subject was presented with one of two possible stimuli and was required to determine if the item had been presented previously.

Second, within the two procedures, there were a number of procedural distinctions which might have differentially influenced retention. In Experiment 1, a trial was not initiated until a subject responded on the rear lever, consequently the intertrial interval, although always greater than $30 \mathrm{sec}$, was variable. In Experiement 2, there was no such "attentional" response requirement and the ITI was fixed at $30 \mathrm{sec}$. Other differences include the nature of the response requirements, the use of a time-out interval for incorrect responses in Experiment 1, and the species and age differences of the sample populations used.

Despite these conceptual and procedural differences, both experiments were consistent in showing a more rapid forgetting function for visual events than for auditory events. In addition, both experiments demonstrated that this difference in retention occurred in spite of the fact that visual and auditory signals led to equal retention at 0 -sec delay intervals. Thus, the results cannot be attributable to differences in discrimination difficulty between the two signals.

Few investigators have demonstrated differential forgetting functions that can be attributed to specific aspects of a to-be-remembered event. Maki, Moe, and Bierly (1977), comparing pigeons' memory of 
stimuli, responses, and reinforcers, found that various interference procedures disrupted the memory of these events to an equal degree, suggesting that similar mechanisms are responsible for the retention of these items. Similarly, Wilkie (1978) showed that the absence of food is as memorable as the presence of food. However, Wilkie (1978) did suggest the possibility that retention of food reinforcers might be more durable than the retention of color or form stimuli. This hypothesis was tentatively based on the noticeable differences across studies of the degree to which food and visual events can effectively control responding over long delay intervals.

Retention has been shown, however, to be influenced by the duration of the stimulus event. Nelson and Wasserman (1978) and Shimp and Moffit (1977), using a Konorski-type procedure, reported that forgetting of visual samples was inversely related to the duration of stimulus exposure. Roberts and Grant (1974) reported the same effect, again with pigeons, for color samples within a DMTS task.

An even stronger example of memory content influencing retention is found in a study by Farthing, Wagner, Gilmour, and Waxman (1977). In this study, it was shown that, although line-tilt and color samples produced equivalent discrimination performance at 0 -sec delay intervals, line orientation showed a steeper forgetting function than color. In addition, increases in stimulus exposure facilitated retention to a greater degree for color stimuli than for line orientation. These authors suggest that retention for stimulus events at longer than 0 -sec delays is dependent upon the subjects' engaging in rehearsal coding strategies. Differences in the retention of specific events may, therefore, result from less efficient encoding strategies for some stimulus dimensions than for others.

For the present experiments, the Farthing et al. (1977) hypothesis would suggest that rats are capable of encoding auditory events more efficiently than visual events. Based on our knowledge of the rats' limited visual capacity, this hypothesis, at present, appears a likely explanation. An interesting outgrowth of this notion would suggest that an auditory event may serve as a better retrieval cue than a visual event for the rat. Whether or not this prediction will be validated awaits further experimentation.

\section{REFERENCE NOTES}

1. Steinert, P., Fallon, D., \& Wallace, J. Matching-to-sample in goldfish (Carassius auratus). Paper presented at the Psychonomic Society meetings, St. Louis, 1976.

\section{REFERENCES}

Carter, D. E., \& Eckerman, D. A. Symbolic matching by pigeons: Rate of learning complex discriminations predicted from sample discriminations. Science, 1975, 187, 662-664.

Cohen, L. R., Looney, T. A., Brady, J. H., \& Aucella, A. F. Differential sample response schedules in the acquisition of conditional discriminations by pigeons. Journal of the Experimental Analysis of Behavior, 1976, 26, 301-304.

Crowder, R. G. Principles of learning and memory. Hillsdale, N.J: Erlbaum, 1976.

D'Aмато, M. R. Delayed matching and short term memory in monkeys. In G. H. Bower (Ed.), The psychology of learning and motivation: Advances in research and theory (Vol. 3). New York: Academic Press, 1973.

D'Amato, M. R., \& Worsham, R. W. Retrieval cues and shortterm memory in Capuchin monkeys. Journal of Comparative and Physiological Psychology, 1974, 86, 274-282.

Eckerman, D. A. Generalization and response mediation of a conditional discrimination. Journal of the Experimental Analysis of Behavior, 1970, 13, $301-316$.

Farthing, G. W., Wagner, J. M., Gilmour, S., \& Waxman H. M. Short-term memory and information processing in pigeons. Learning and Motivation, 1977, 8, 520-532.

Gordon, W. C., Brennan, M. J., \& Schlesinger, J. L. The interaction of memories in the rat: Effects on short-term retention performance. Learning and Motivation, 1976, 7, 406-417.

Herman, L. M., \& Gordon, J. A. Auditory delayed matching in the bottlenose dolphin. Journal of the Experimental Analysis of Behavior, 1974, 21, 19-26.

Hunter, W. S. The delayed reaction in animals and children. Behavior Monographs, 1913, 2, 1-86.

KepPel, G., \& UnderwOod, B. Proactive inhibition in shortterm retention of single items. Journal of Verbal Learning and Verbal Behavior, 1962, 1, 153-161.

Konorski, J. A new method of physiological investigation of recent memory in animals. Bulletin de l'Academie Polonaise des Sciences, 1959, 7, 115-117.

Maki, W. S., Jr., Moe, J. C., \& Bierly, C. M. Short-term memory for stimuli, responses and reinforcers. Journal of Experimental Psychology: Animal Behavior Processes, 1977, 3, 156-177.

Nelson, K. R., \& Wasserman, E. A. Temporal factors influencing the pigeon's successive matching-to-sample performance: Sample duration, intertrial interval, and retention interval. Journal of the Experimental Analysis of Behavior, 1978, 30, 153-162.

RoBerts, W. A. Spaced repetition facilitates short-term retention in the rat. Journal of Comparative and Physiological Psychology, 1974, 86, 164-171.

Roberts, W. A., \& Grant, D. S. Short-term memory in the pigeon with presentation time precisely controlled. Learning and Motivation, 1974, 5, 393-408.

Roberts, W. A., \& Grant, D. S. Studies of short-term memory in the pigeon using the delayed matching to sample procedure. In D. L. Medin, W. A. Roberts, \& R. T. Davis (Eds.), Processes of animal memory. Hillsdale, N.J: Erlbaum, 1976.

Shimp, C. P., \& Moffit, M. Short-term memory in the pigeon: Delayed-pair-comparison procedures and some results. Journal of the Experimental Analysis of Behavior, 1977, 28, 13-25.

SPE AR, N. E. The processing of memories: Forgetting and retention. Hillsdale, N.J: Erlbaum, 1978.

Wallace, J. E., Krauter, E. E., \& Campbell, B. A. Animal models of declining memory in the aged: Short-term retention in the rat as a function of age. Journal of Gerontology, in press.

Wasserman, E. A. Successive matching-to-sample in the pigeon: Variations on a theme by Konorski. Behavior Research Methods \& Instrumentation, 1976, 8, 278-282.

WILkIE, D. M. Delayed symbolic matching to sample in the pigeon. Psychological Record, 1978, 28, 463-469.

Worsham, R. W., \& D'Amato, M. R. Ambient light, white noise, and monkey vocalization as sources of interference in visual short-term memory of monkeys. Journal of Experimental Psychology, 1973, 99, 99-105.

(Received for publication January 8, 1979; revision accepted May 9,1979 .) 